\title{
Technical note: Designing and analyzing quantitative factorial experiments ${ }^{1}$
}

\author{
N. R. St-Pierre ${ }^{2}$ and W. P. Weiss \\ Department of Animal Sciences, The Ohio State University, Columbus 43210
}

\begin{abstract}
The response of a biological process to various factors is generally nonlinear, with many interactions among those factors. Although meta-analyses of data across multiple studies can help in identifying and quantifying interactions among factors, missing latent variables can result in serious misinterpretation. Eventually, all influential factors have to be studied simultaneously in one single experiment. Because of the curvature of the expected response and the presence of interactions among factors, the size of experiments grows very large, even when only 3 or 4 factors are fully arranged. There exists a class of experimental designs, named central composite designs (CCD), that considerably reduces the number of treatments required to estimate all the terms of a second-order polynomial equation without any loss of efficiency compared with the full factorial design. The objective of this technical note is to explain the construction of a CCD and its statistical analysis using the Statistical Analysis System. In short, a CCD consists of $2^{k}$ treatment points (a first-order factorial design, where $k$ represents the number of factors), augmented by at least one center point and $2 \times k$ axial treatments. For 3 factors, the resulting design has 16 treatment points, compared with 27 for a full factorial design. For 4 factors, the CCD has 25 treatment points, compared with 81 for a full factorial design. The CCD can be made orthogonal (no correlation between parameter estimates) or rotatable (the variance of the estimated response is a function only of the distance from the design center and not the direction) by the location of the axial treatments. In spite of the reduced number of treatments compared with a full $3^{k}$ factorial, the CCD is relatively efficient in estimation of the quadratic and interaction terms. Blocking of experimental units is often desirable and is sometimes
\end{abstract}

Received March 31, 2009.

Accepted May 26, 2009.

${ }^{1}$ Salaries and research support were provided by state and federal funds appropriated to the Ohio Agricultural Research and Development Center, The Ohio State University. This project was supported by National Research Initiative Competitive Grant no. 2005-35206-15263 from the USDA Cooperative State Research, Education, and Extension Service (Washington, DC). Manuscript number 7-09AS.

${ }^{2}$ Corresponding author: st-pierre.8@osu.edu required. Randomized block designs for CCD are found in some statistical design textbooks. The construction of incomplete, balanced (or near-balanced) designs for CCD experimental layouts is explained using an example. The Statistical Analysis System statements used to analyze a CCD, to identify the significant parameters in the polynomial equation, and to produce parameter estimates are presented and explained.

Key words: factorial experiment, central composite design, randomized incomplete block design, mixed model

Experimental research generally follows a classic path. Initially, experiments are designed to study the effects of 1 or 2 factors while holding all other factors constant. This is the domain of the 1 -way and 2 -way treatment designs. These experiments help uncover the fundamental processes involved, but quantification of the relationships remains problematic because of the implicit linearity in the resulting estimated relationships. The difficulty in identifying and quantifying interaction effects when one is pooling results across many experiments, such as in quantitative meta-analyses (StPierre, 2000), is often misjudged. Any hidden, latent variable can bias all such analyses and their interpretation. Eventually, scientists need to design experiments in which multiple factors are considered simultaneously to quantify the curvature (nonlinearity) in relationships as well as the interactions among factors. This is especially true for certain components of nutritional systems such as energy and microbial production, for which it is important that robust quantitative relationships be developed, thus requiring the inclusion of curvature and interaction terms. Approximating curvature through quadratic polynomials necessitates at least 3 levels for each factor. If a conventional factorial arrangement of treatments is used, the number of experimental treatments required grows geometrically with the number of factors considered. For 2 factors, the resulting $3 \times 3$ factorial requires 9 different treatments. For 3 factors, the $3 \times 3 \times 3$ factorial arrangement requires 27 treatments; $k$ factors result in $3^{k}$ treatments. In addition to the enormous and generally unpractical number of treatments involved, many experimental designs cause parameter estimates (the regression coefficients in a 


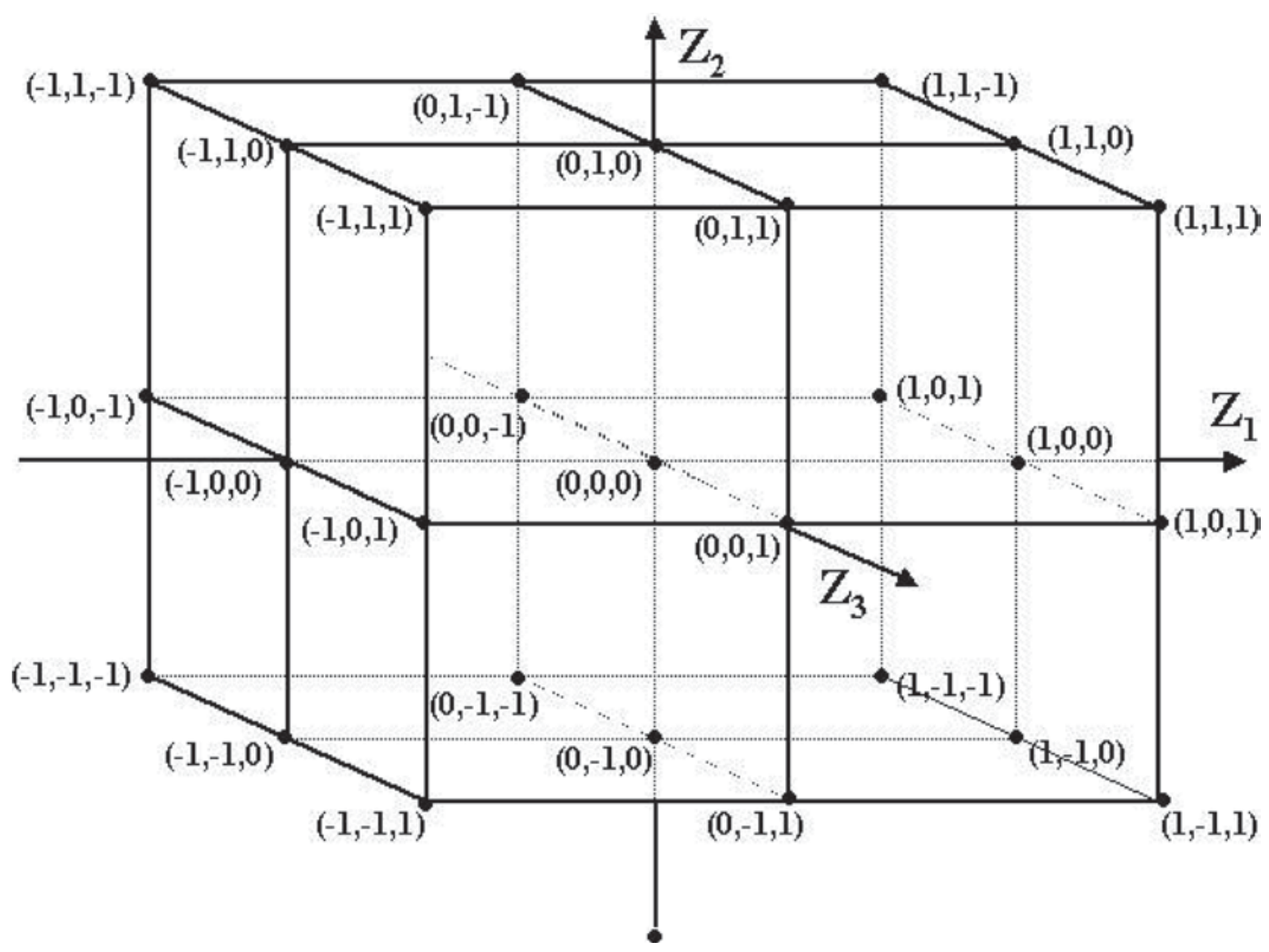

Figure 1. Three-dimensional representation of a factorial treatment arrangement for 3 factors $(k=3$; number of treatments $=27)$. The 3 factors $\left(\mathrm{Z}_{1}, \mathrm{Z}_{2}, \mathrm{Z}_{3}\right)$ are coded so that their minima, central values, and maxima take values of $-1,0$, and 1 , respectively.

polynomial equation) that are correlated, thus lowering the power of the experiment. For example, the coefficients for the squared terms of a polynomial function are estimated with relatively low precision with $3^{k}$ factorial experiments (Box and Wilson, 1951). There exists a class of experimental designs, named central composite designs (CCD), that can considerably reduce the number of treatments required to estimate all parameters in the model while producing parameter estimates that can be made independent (i.e., orthogonal) of each other (Myers et al., 2009). Unfortunately, the CCD has received little attention in the statistical literature related to biology and agriculture; most textbooks on statistical designs in biology do not even mention CCD. Our objectives are to explain how a CCD works, how to construct a CCD with incomplete blocks, and how to conduct the analysis of biological data from CCD experiments using SAS.

\section{The Structure of a CCD}

We start with a simple example of a 3 -factor experiment. The objective is to estimate the curvilinear response surface of a dependent variable $\mathrm{Y}$ using a quadratic polynomial function of 3 factors, $\mathrm{X}_{1}, \mathrm{X}_{2}$, and $\mathrm{X}_{3}$. The statistical model is

$$
\begin{gathered}
Y_{i}=b_{0}+b_{1} X_{1}+b_{2} X_{1}^{2}+b_{3} X_{2}+b_{4} X_{2}^{2}+b_{5} X_{3} \\
+b_{6} X_{3}^{2}+b_{7} X_{1} X_{2}+b_{8} X_{1} X_{3}+b_{9} X_{2} X_{3}+e_{i}
\end{gathered}
$$

where $Y_{i}$ are the values of the dependent variable, $b_{j}$ are the regression parameters to be estimated, and $\mathrm{e}_{\mathrm{i}}$ are the residual errors, assumed independent, normal, with a mean of zero and a variance of $\sigma_{e}^{2}$. The treatment design for a conventional $3 \times 3 \times 3$ factorial experiment is illustrated in Figure 1. A total of 27 treatments is required to maintain a balanced design and independent parameter estimates. Least squares (and maximum likelihood) estimates of the $b_{i}$ are easily calculated (Draper and Smith, 1998).

Designs for fitting second-order response surfaces such as in equation [1] must involve at least 3 levels of each variable so that all the coefficients in the model can be estimated. The CCD is a first-order factorial design augmented with additional points to allow the estimation of the coefficients of a second-order surface. A 3-factor CCD is illustrated in Figure 2. With 3 factors, the CCD requires only 15 individual treatments to provide estimates of all 10 parameters in equation [1], compared with 27 treatments for the full $3 \times 3 \times 3$ factorial. With a CCD, it is often desirable to replicate 
the center point (or treatment) to attain certain design properties (orthogonality, rotatability) and to allow the splitting of the residual error into lack-of-fit and pure error components. These are discussed below.

In Figure 2, the first 8 points located at the vertices (corners) are the usual factorial points for fitting a firstorder model. The ninth point is the center point of the design, which, as we just mentioned, is often repeated $n_{2}$ times $\left(n_{2}\right.$ is the number of replicates of the center point). The 6 remaining points are called the axial points. Notice that with a CCD, each independent variable is actually measured at 5 levels: $-\alpha,-1,0,1,+\alpha$. The choice of $\alpha$ results in different design properties, such as orthogonality and rotatability. The design can be made orthogonal, resulting in uncorrelated estimates of the response model coefficients. Table 1 reports the values of $\alpha$ for an orthogonal CCD with a different number of factors $(k)$ when $n_{2}=1$. The design can also be made rotatable, where the variance of the estimated response is a function only of the distance from the center and not the direction (Myers and Montgomery, 1995). Table 1 also reports the values of $\alpha$ to obtain a rotatable CCD when $n_{2}=1$.

The CCD can be compared with the $3^{k}$ factorial design on the basis of efficiency in estimating particular coefficients in the response model. Many efficiency
Table 1. Values of $\alpha$ for an orthogonal or rotatable central composite design (CCD) if a full-factorial design is used with a single center point

\begin{tabular}{lcc}
\hline & \multicolumn{2}{c}{$\alpha^{2}$} \\
\cline { 2 - 3 }$k^{1}$ & Orthogonal & Rotatable \\
\hline 2 & 1.000 & 1.414 \\
3 & 1.216 & 1.682 \\
4 & 1.414 & 2.000 \\
5 & 1.596 & 2.378 \\
6 & 1.761 & 2.828 \\
7 & 1.910 & 3.364 \\
8 & 2.045 & 4.000 \\
\hline
\end{tabular}

${ }^{1} k$ is the number of factors.

${ }^{2}$ In a CCD, $\alpha$ represents the coded distance between the maximum level of a factor and its central (median) value (see Figure 2).

criteria have been developed, such as A-, D-, and G-optimality criteria (Myers and Montgomery, 1995), each emphasizing a different desirable property of designs. An efficiency criterion was developed to simultaneously take into consideration the precision with which coefficients are estimated and the number of experimental treatments required (Keifer, 1975). Table 2 gives the relative efficiencies for estimating the mixed and pure quadratic coefficients for the orthogonal CCD compared with the $3^{k}$ factorial design. In this table, coefficients greater than 1.0 indicate that the CCD has a greater

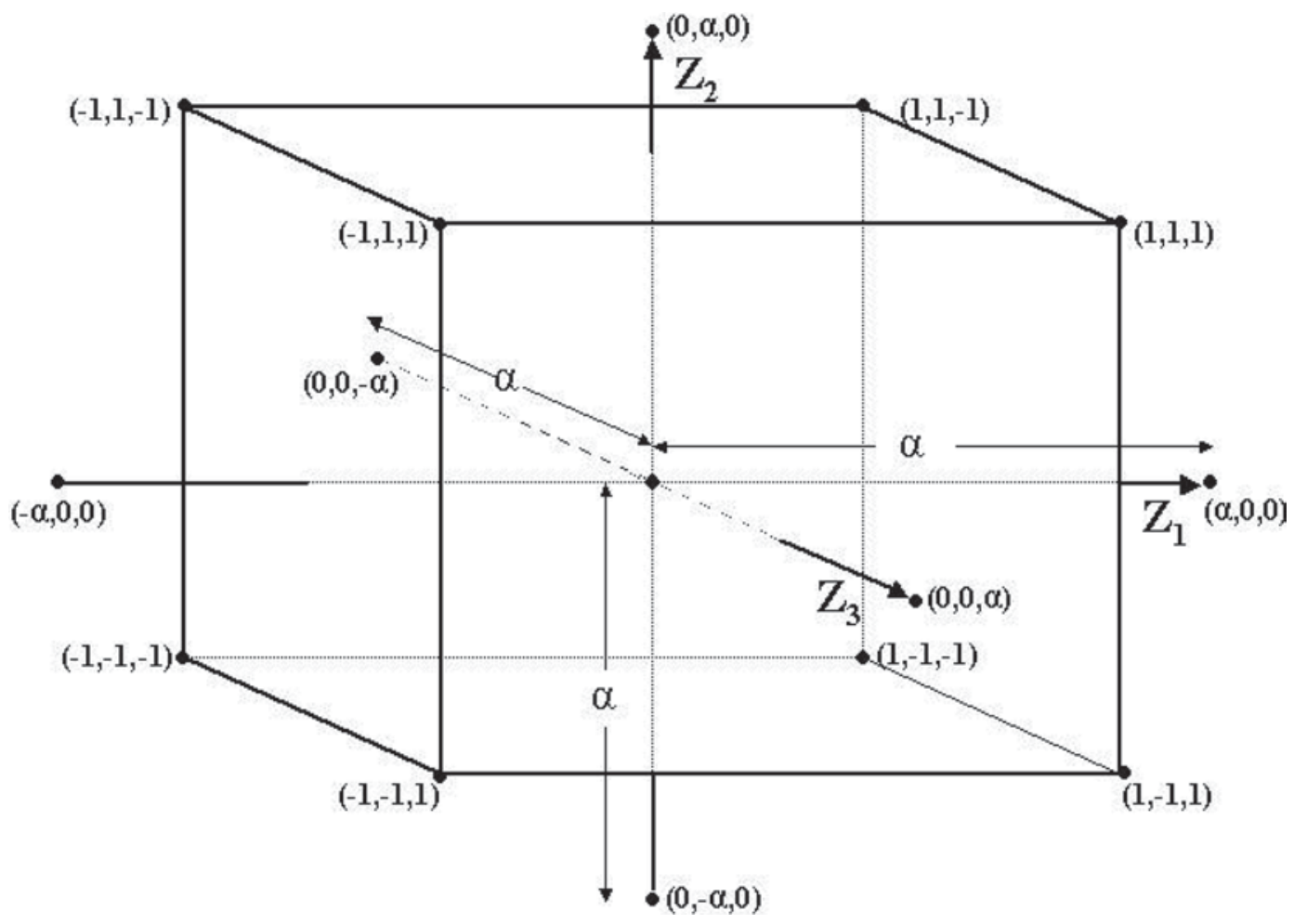

Figure 2. Three-dimensional representation of a central composite design for 3 factors $(k=3$; number of treatments $=15)$. The 3 factors $\left(\mathrm{Z}_{1}, \mathrm{Z}_{2}, \mathrm{Z}_{3}\right)$ are coded levels of the 3 factors. Note that the minima and maxima take values of $-\alpha$ and $\alpha$, respectively. 
Table 2. Relative efficiencies of orthogonal central composite designs (CCD) compared with $3^{k}$ factorials ${ }^{1}$

\begin{tabular}{lrrrrc}
\hline Item & $k=2$ & $k=3$ & $k=4$ & $k=5^{2}$ & $k=6^{2}$ \\
\hline $\mathrm{b}_{\text {int }}{ }_{3}$ & 1.00 & 1.00 & 1.00 & 1.00 & 1.00 \\
$\mathrm{~b}_{\text {squ }}$ & 1.00 & 1.07 & 1.00 & 1.43 & 1.62 \\
\hline
\end{tabular}

${ }^{1} k$ is the number of factors.

${ }^{2}$ Denotes CCD containing $1 / 2$ fraction of a $2^{k}$ plus appropriate axial points and center points.

${ }^{3} b_{\text {int }}$ and $b_{\text {squ }}$ are the regression coefficients for the interaction terms (mixed coefficients) and for the squared terms (pure quadratic coefficients) in the polynomial equation, respectively.

efficiency than the factorial design (i.e., 1.07 indicated a $7 \%$ greater efficiency of the CCD). Clearly, according to this efficiency criterion, the CCD is always at least as efficient as the $3^{k}$ factorial. Other efficiency criteria would yield different results, but in general, CCD is quite efficient (Myers and Montgomery, 1995).

\section{Blocking of Treatments}

The CCD is, in fact, a treatment arrangement that can be arranged in a variety of experimental designs. The randomized incomplete block design (RIBD) is generally an attractive statistical design for CCD to decrease the number of treatments needed. Some arrangements of CCD in RIBD have been given by Box and Hunter (1957), and the methods to conduct statistical analysis when blocks are considered to have a fixed effect have been fully described (Cochran and Cox, 1957). The CCD has frequently been used in the design of industrial experiments, in which blocks are more appropriately considered as having a fixed effect. In biological experiments, however, blocks are often the outcome of a random process; their effects should be considered random (Mead and Pike, 1975). Using a mixed model with random blocks allows the recovery of interblock as well as intrablock information (Khuri, 1992). Regardless of the type of block effects, it is always desirable that the design be divided into blocks in such a manner that the block effect does not affect the estimates of the polynomial terms in the model. That is, blocking should be orthogonal or as close to it as possible. Some design plans that ensure block orthogonality are found in numerous textbooks such as that by Cochran and Cox (1957). Often a layout has to be designed to accommodate specific restrictions imposed by a given experiment because of facilities, costs, animal availability, and so on. An example of such a construction is presented in the next section.

\section{Data Analysis Using SAS}

The analysis of CCD data using the Statistical Analysis System (SAS) is explained using a recent trial (Weiss, W. P., N. R. St-Pierre, and L. B. Willett, unpublished data; Weiss, W. P., L. B. Willett, N. R. St-Pierre, D. C. Borger, T. R. McKelvey, and D. J. Wyatt, unpublished data) as an example. Prior research identified numerous nutritional factors influencing the fraction of total manure $\mathrm{N}$ that volatilizes as $\mathrm{NH}_{3}$. In nearly all instances, prior research had focused on one factor at a time. Our interest was in the quantitative estimation of the response of several production variables including manure $\mathrm{NH}_{3}$ volatilization to the levels of 3 dietary factors: starch (\% of DM), MP (\% of DM), and alfalfa (\% of forage DM). A full-factorial arrangement that would allow the estimation of quadrature would have required $3^{3}=27$ different diets. In contrast, a CCD required $2^{3}+(2 \times 3)+1=15$ different diets. The experiment involved total collection of feces and urine. The variance between cows for numerous metabolic variables is substantial. Thus, a crossover-type design was highly desirable to minimize the number of cows and total collections to be done. A full Latin square was clearly out of the question: using a multiple of 15 cows over 15 collection periods of 3 wk each would

Table 3. Treatment layout for a 3-factor orthogonal central composite design (CCD) with $n_{2}=4$ central points $^{1}$

\begin{tabular}{lccc}
\hline & \multicolumn{3}{c}{ Factor $^{2}$} \\
\cline { 2 - 4 } Treatment & $\mathrm{X}_{1}$ & $\mathrm{X}_{2}$ & $\mathrm{X}_{3}$ \\
\hline 1 & 1 & 1 & 1 \\
2 & 1 & -1 & -1 \\
3 & -1 & 1 & -1 \\
4 & -1 & -1 & 1 \\
$5^{3}$ & 0 & 0 & 0 \\
$6^{3}$ & 0 & 0 & 0 \\
7 & 1 & 1 & -1 \\
8 & 1 & -1 & 1 \\
9 & -1 & 1 & 1 \\
10 & -1 & -1 & 0 \\
$11^{3}$ & 0 & 0 & 0 \\
$12^{3}$ & 0 & 0 & 0 \\
13 & -1.4142 & 0 & 0 \\
14 & 1.4142 & -1.4142 & 0 \\
15 & 0 & 1.4142 & 0 \\
16 & 0 & 0 & -1.4142 \\
17 & 0 & 0 & 1.4142 \\
18 & 0 & & 1 \\
\hline
\end{tabular}

${ }^{1}$ Coefficients in the table are the coded levels for each of the three factors $\left(\mathrm{X}_{1}, \mathrm{X}_{2}, \mathrm{X}_{3}\right)$.

${ }^{2}$ In the example used in the text, $\mathrm{X}_{1}, \mathrm{X}_{2}$, and $\mathrm{X}_{3}$ refer to the percentage of alfalfa (A), the percentage of starch (S), and the percentage of MP $(\mathrm{P})$ in the diet, respectively. One unit level in $\mathrm{X}_{1}(\mathrm{~A})$ corresponded to $17.68 \%$ of alfalfa (\% of forage DM); 1 unit of $\mathrm{X}_{2}$ (S) corresponded to $2.83 \%$ starch (\% of DM), and 1 unit of $\mathrm{X}_{3}(\mathrm{P})$ corresponded to $1.6 \%$ of MP (\% of DM). Treatments are centered (coded value of 0 ) at $50 \%$ alfalfa, $26 \%$ starch, and $10.4 \%$ MP. Thus, treatment 1 corresponds to a diet with $67.7 \%$ alfalfa, $28.8 \%$ starch, and $11.5 \%$ MP.

${ }^{3}$ Treatments 5, 6, 11, and 12 are the same treatment; the different treatment numbers simply reflect replication of the center point treatment. 
have exceeded the life expectancy of too many cows, if not that of the authors themselves. In addition, our research facilities allow for a maximum of 6 cows at a time in the metabolism stalls. This imposed a maximum block size of 6 cows. Prior experience with full fecal and urinary collection experiments indicated that 3 periods of 3 wk each resulted in a very good state of animal welfare with a minimal loss of data. Thus, we had to set multiple blocks of 6 cows, each with 3 periods of collection. That is, each cow would go through a sequence of 3 diets out of the total 15 diets. To ensure balance and orthogonality, the number of treatments must be a multiple of the number of cows per block. In a CCD, this is achieved by adding measurements at the center point. In our case, this meant that the center point diet would be repeated 4 times (i.e., $n_{2}=4$ ), for a total of 18 treatments, with the understanding that 4 of these treatments would be identical (i.e., treatments $5,6,11$, and 12 in Table 3).

In the design of this experiment, orthogonality of the parameter estimates (the $b_{i}$ in equation [1]) is desirable because nonsignificant terms are to be removed from the equation and orthogonality ensures that the tests on the estimates are independent of each other. Thus, issues associated with sequential tests are avoided. Because $n_{2}=4$, Table 1 cannot be used to determine the correct value for $\alpha$. It has to be calculated as follows (Myers and Montgomery, 1995). Let $F$ be the number of factorial treatment points (i.e., $2^{k}$ if a complete factorial design is used, where $k$ is the number of factors), and $T=2 k+n_{2}$ (recall that $n_{2}$ is the number of center points). First, calculate

$$
Q=\left[(F+T)^{1 / 2}-F^{1 / 2}\right]^{2} .
$$

Then $\alpha$ is given by

$$
\alpha=(\mathrm{Q} F / 4) 1 / 4 \text {. }
$$

In our application, $k=3, F=8, T=10$, and $Q=2$; therefore, $\alpha=1.4142$. The resulting treatment layout is reported in Table 3 .

Because no such experimental design exists in the literature, we constructed a crossover RIBD, which could also be described as a replicated, incomplete (truncated) Latin square with 6 blocks, each of 6 cows and 3 periods, and with 18 treatments. To ensure near orthogonality and to balance for any possible carryover effects, the following rules had to be followed during its construction:

- Rule 1: Each treatment must be replicated exactly 6 times and appear once in each block.
- Rule 2: Each treatment must appear equally often in each of the 3 periods across blocks.

- Rule 3: Each treatment is followed by another treatment only once.

- Rule 4: Each block is to contain exactly 4 central point treatments (i.e., treatments 5, 6, 11, and 12).

- Rule 5: There must be no more than 2 central point treatments in a given period.

- Rule 6: The frequency of 2 central point treatments in a period must be the same in each of the 3 periods across blocks.

- Rule \%: A central point treatment is not to follow another central point treatment.

- Rule 8: Treatment sequences should minimize the frequency when 1 or more of the 3 factors is either below or above the center point in all 3 periods.

- Rule 9: There must be no sequence in which a factor is at the same level across 3 periods.

To avoid a fastidious manual search, we used a genetic algorithm (St-Pierre and Cobanov, 2006) to identify a layout that matched these rules. The resulting experimental layout is shown in Figure 3.

Factors $\mathrm{X}_{1}, \mathrm{X}_{2}$, and $\mathrm{X}_{3}$ in Table 3 refer to the coded levels of alfalfa (A), starch (S), and MP (P) in the diets. The coding worked as follows. First, minimum and maximum concentrations of each factor were set, based on typical ranges observed in the field, feed cost considerations (e.g., MP maximum), and cow health considerations (e.g., starch maximum). For alfalfa, the minimum and maximum levels were set at 25 and $75 \%$. A 1- $\alpha$ unit of $\mathrm{X}_{1}$ (i.e., A) corresponds to $(75-50)=$ $25 \%$ of alfalfa (\% of forage DM). Recalling that $\alpha=\sqrt{ } 2$ in our application, a coded 1 unit of A corresponds to $25 \div \sqrt{ } 2=17.68 \%$ alfalfa. Thus, the coded levels for $\mathrm{X}_{1}$ (A) in Table 3 correspond to 25.0, 32.3, 50.0, 67.7, and $75.0 \%$ alfalfa in the diet. For starch, the minimum and maximum levels were set at 22 and $30 \%$ of DM, respectively. Thus, 1 unit of S corresponds to $2.83 \%$ starch in diet DM, and the 5 levels of starch used were 22.0, 23.2, 26.0, 28.8, and $30.0 \%$ starch. For MP, the minimum and maximum were set at 8.8 and $12.0 \%$ of DM. Therefore, 1 unit of $\mathrm{P}$ corresponds to 1.1\% MP in diet DM, and the 5 levels of MP used were 8.8, 9.3,10.4, 11.5, and $12.0 \%$ MP. A complete data set is provided for total manure output (kg/cow per day) in the Appendix section (see Figure A1) to allow readers to replicate the calculations and procedures explained in this article. Data are also available as a downloadable Excel spreadsheet (http:// dairy.osu.edu). Readers should note that cow 4137 in block 3 had to be removed from the experiment in period 2 and was replaced by cow 4523 in period 3 . This created 


\begin{tabular}{|c|c|c|c|c|c|c|}
\hline \multicolumn{7}{|l|}{ Block 1} \\
\hline Period & $A$ & $B$ & $\mathrm{C}$ & $\mathrm{D}$ & $E$ & $\mathrm{~F}$ \\
\hline $\mathrm{I}$ & 5 & 4 & 7 & 10 & 13 & 16 \\
\hline II & 1 & 17 & 11 & 14 & 8 & 2 \\
\hline IIII & 18 & 12 & 15 & 9 & 3 & 6 \\
\hline
\end{tabular}

\begin{tabular}{|c|c|c|c|c|c|c|}
\hline \multicolumn{7}{|l|}{ Block 2} \\
\hline Period & $A$ & B & C & $\mathrm{D}$ & $E$ & $\mathrm{~F}$ \\
\hline 1 & 11 & 17 & 14 & 12 & 7 & 9 \\
\hline II & 1 & 13 & 5 & 15 & 4 & 18 \\
\hline III & 10 & 2 & 3 & 8 & 16 & 6 \\
\hline
\end{tabular}

\begin{tabular}{|c|c|c|c|c|c|c|}
\hline \multicolumn{6}{|l|}{ Block 3} & \\
\hline Period & $A$ & $B$ & $\mathrm{C}$ & $\mathrm{D}$ & $E$ & $\mathrm{~F}$ \\
\hline $\mathrm{I}$ & 10 & 1 & 3 & 15 & 18 & 12 \\
\hline II & 2 & 6 & 14 & 4 & 11 & 16 \\
\hline III & 13 & 17 & 7 & 5 & 8 & 9 \\
\hline
\end{tabular}

\begin{tabular}{|c|c|c|c|c|c|c|}
\hline \multicolumn{7}{|l|}{ Block 4} \\
\hline Period & $A$ & $B$ & $\mathrm{C}$ & $\mathrm{D}$ & $E$ & $\mathrm{~F}$ \\
\hline I & 13 & 6 & 1 & 16 & 3 & 9 \\
\hline II & 5 & 2 & 17 & 12 & 15 & 7 \\
\hline III & 4 & 14 & 10 & 8 & 11 & 18 \\
\hline
\end{tabular}

\begin{tabular}{|c|c|c|c|c|c|c|}
\hline \multicolumn{6}{|l|}{ Block 5} & \\
\hline Period & $A$ & $B$ & $\mathrm{C}$ & D & $E$ & $\mathrm{~F}$ \\
\hline 1 & 18 & 8 & 15 & 5 & 2 & 11 \\
\hline II & 4 & 6 & 3 & 13 & 9 & 16 \\
\hline III & 1 & 14 & 10 & 7 & 12 & 17 \\
\hline
\end{tabular}

\begin{tabular}{|ccccccc|}
\hline Block 6 & \multicolumn{7}{c|}{ Cows } \\
\cline { 2 - 7 } Period & A & B & C & D & E & F \\
\hline I & 2 & 14 & 6 & 8 & 4 & 17 \\
II & 12 & 7 & 18 & 13 & 10 & 3 \\
III & 9 & 1 & 16 & 11 & 15 & 5 \\
\hline
\end{tabular}

Figure 3. Experimental layout for a 3-period crossover, randomized, balanced incomplete block design with 6 blocks of 6 cows and 18 treatments. Cells in each block refer to the treatment numbers of the central composite treatment design of Table 3 . Cows and periods are nested within blocks (i.e., cow $\mathrm{A}$ in block 1 is different from cow $\mathrm{A}$ in block 2; period I in block 1 is different from period I in block 2).

a slight imbalance in the data, with the consequence that the coefficient estimates in the polynomial equations were not perfectly orthogonal.

The statistical model for data from this experiment includes the fixed effects of A, S, P, their squared terms, and their single interactions terms (i.e., equation [1]), whereas blocks, period within blocks, and cows within blocks were considered random effects. This model is solved using the MIXED procedure (SAS Institute, 2004). In a first step, the complete model is fitted using the coded values (A, S, P) for the 3 factors (output is in Figure 4):
PROC MIXED COVTEST;

CLASSES Block Period Cow;

MODEL Manure $=S \mathrm{P} A$

$\mathrm{S} * \mathrm{~S} \quad \mathrm{P} * \mathrm{P} \quad \mathrm{A} * \mathrm{~A}$

$\mathrm{S} * \mathrm{P} \quad \mathrm{S} * \mathrm{~A} \quad \mathrm{P} * \mathrm{~A} / \mathrm{CORRB}$ DDFM $=\mathrm{KR}$;

RANDOM Block Period(Block) Cow(Block);

RUN ;

The CORRB option in the MODEL statement calculates and reports the approximate correlation matrix of the fixed-effects parameter estimates. If the design were perfectly orthogonal, all its off-diagonal elements would be equal to zero (i.e., the correlation between all pairs of estimates would be equal to zero). Because of the loss and replacement of $1 \mathrm{cow}$, the design was no longer perfectly orthogonal, but the approximate correlation matrix showed that all correlations were, in fact, very small (all were less than $|0.06|$ and most were less than $|0.01|)$. Figure 4 presents the SAS output relevant to the fixed effects in the model. An orthogonal design implies that the test of a given effect is completely independent of the other effects. That is, the test on the linear effect of S (starch) is completely independent of whether $\mathrm{S}^{2}$ is included in the model. Our design was

\begin{tabular}{|l|r|r|r|r|}
\hline \multicolumn{5}{|c|}{ Type 3 Tests of Fixed Effects } \\
\hline Effect & $\begin{array}{r}\text { Num } \\
\text { DF }\end{array}$ & $\begin{array}{r}\text { Den } \\
\text { DF }\end{array}$ & F Value & Pr > F \\
\hline S & 1 & 66.4 & 6.72 & 0.0117 \\
\hline $\mathbf{P}$ & 1 & 66.1 & 0.01 & 0.9128 \\
\hline $\mathbf{A}$ & 1 & 66.9 & 15.06 & 0.0002 \\
\hline $\mathbf{S} * \mathbf{S}$ & 1 & 61.9 & 0.19 & 0.6614 \\
\hline $\mathbf{P} * \mathbf{P}$ & 1 & 61.9 & 0.06 & 0.8021 \\
\hline $\mathbf{A} * \mathbf{A}$ & 1 & 65.3 & 0.18 & 0.6690 \\
\hline $\mathbf{S} \mathbf{P}$ & 1 & 62.9 & 0.25 & 0.6155 \\
\hline $\mathbf{S} * \mathbf{A}$ & 1 & 70.9 & 0.96 & 0.3307 \\
\hline $\mathbf{P} * \mathbf{A}$ & 1 & 65.2 & 0.99 & 0.3245 \\
\hline
\end{tabular}

Figure 4. Statistical tests of the fixed-effect factors reported by the MIXED procedure (SAS Institute, 2004) for the coded factors, where $\mathrm{S}$ is the coded level of starch, $\mathrm{P}$ is the coded level of MP, and A is the coded level of alfalfa. The Num DF and Den DF columns report the numerator and denominator degrees of freedom used to calculate the probability $(\mathrm{Pr}>\mathrm{F})$ of the $F$-statistics (F Value) under the null hypothesis that the effect is null. 


\begin{tabular}{|l|c|c|r|r|}
\hline \multicolumn{5}{|c|}{ Type 3 Tests of Fixed Effects } \\
\hline Effect & $\begin{array}{c}\text { Num } \\
\text { DF }\end{array}$ & $\begin{array}{c}\text { Den } \\
\text { DF }\end{array}$ & F Value & Pr > F \\
\hline Alf & 1 & 72.4 & 16.77 & 0.0001 \\
\hline Starch & 1 & 72.8 & 7.39 & 0.0082
\end{tabular}

\begin{tabular}{|l|r|r|r|r|r|}
\hline \multicolumn{7}{|c|}{ Estimates } \\
\hline Label & Estimate & $\begin{array}{c}\text { Standard } \\
\text { Error }\end{array}$ & DF & t Value & $\operatorname{Pr}>|\mathbf{t}|$ \\
\hline Intercept & 91.9574 & 8.8597 & 76.3 & 10.38 & $<.0001$ \\
\hline Alfalfa & 0.2058 & 0.05026 & 72.4 & 4.10 & 0.0001 \\
\hline Starch & -0.8545 & 0.3144 & 72.8 & -2.72 & 0.0082 \\
\hline
\end{tabular}

Figure 5. Statistical tests and parameter estimates of the fixedeffect factors reported by the MIXED procedure (SAS Institute, 2004) for the final model with uncoded factor effects. The resulting equation is Manure $(\mathrm{kg} / \mathrm{d})=92.0( \pm 8.86)+0.206( \pm 0.050) \times$ Alfalfa $(\%$ of forage DM $)-0.855( \pm 0.314) \times$ Starch $(\%$ of DM). Num DF and Den $\mathrm{DF}$ represent numerator and denominator degrees of freedom, respectively. Alf $=$ alfalfa.

not perfectly orthogonal, but the correlations between parameter estimates were so small that the effects on the standard error of estimates and, consequently, the statistical test on each estimate were negligible.

Because of the factor orthogonality (or near-orthogonality), it is not necessary to proceed through a questionable sequence in which insignificant terms would be iteratively removed from the model. For example, the prediction of manure excretion must involve only the linear effects of starch and alfalfa levels in the diet (Figure 4). All other fixed effects can be removed simultaneously from the model, and the remaining terms can be expressed in their natural scales, as opposed to the coded ones. The statements (SAS Institute, 2004) to achieve this are as follows (output is shown in Figure 5):

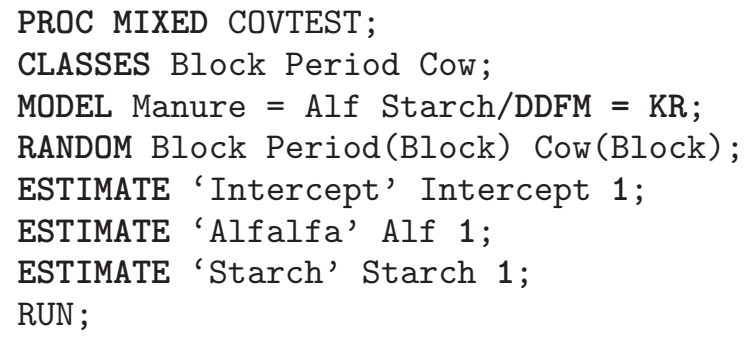

Figure 5 reports the results. Because the analysis is now conducted in the natural scales as opposed to the coded ones, the design is no longer orthogonal and tests on the fixed effects are no longer of interest; parameter estimates and their standard errors are. In our manure example, the resulting prediction equation can be reported as follows:

$$
\begin{gathered}
\text { Manure }(\mathrm{kg} / \mathrm{d})=92.0( \pm 8.86)+0.206( \pm 0.050) \\
\times \text { Alfalfa }(\% \text { of forage } \mathrm{DM})-0.855( \pm 0.314) \\
\times \text { Starch }(\% \text { of } \mathrm{DM}) .
\end{gathered}
$$

Each percentage unit increase in alfalfa concentration results in an expected increase of $0.21 \mathrm{~kg} / \mathrm{d}$ in manure excretion. Likewise, each percentage unit increase in starch concentration results in an expected decrease of $0.8 \mathrm{~kg} / \mathrm{d}$ in manure excretion.

The treatment replications at the center point can be used to partition the residual standard error into a lack-of-fit and pure error terms (Cochran and Cox, 1957). The lack-of-fit test determines whether the fitted quadratic function is a reasonable approximation to the true but unknown relationship.

In this example, the use of a CCD reduced the number of cow-periods from 186 to 112 while maintaining power and the ability to identify all the parameters independently in the quadratic function. The use of a CCD allowed us to conduct an experiment that essentially would be impossible to conduct using standard experimental designs because of limits on cow availability, facility limitations, and economic considerations. Because, in this type of experiment, most of the costs are directly related to the number of cow-periods, the use of a CCD reduced the experimental costs by approximately $40 \%$ compared with a full-factorial treatment design.

\section{REFERENCES}

Box, G. E. P., and J. S. Hunter. 1957. Multifactor experimental designs. Ann. Math. Stat. 28:195-241.

Box, G. E. P., and K. B. Wilson. 1951. On the experimental attainment of optimum conditions. J. R. Stat. Soc., B 13:1-45.

Cochran, W. G., and G. M. Cox. 1957. Experimental Designs. John Wiley and Sons Inc., New York.

Draper, N. R., and H. Smith. 1998. Applied Regression Analysis. 3rd ed. John Wiley and Sons Inc., New York.

Keifer, J. 1975. Optimum design: Variation in structure and performance under change of criterion. Biometrika 62:277-288.

Khuri, A. I. 1992. Response surface models with random block effects. Technometrics 34:26-37.

Mead, R., and D. J. Pike. 1975. A review of response surface methodology from a biometric viewpoint. Biometrics 31:803851.

Myers, R. H., C. Montgomery, and C. M. Anderson-Cook. 2009. Response Surface Methodology. 3rd ed. Wiley-Interscience, New York, NY.

Myers, R. H., and D. C. Montgomery. 1995. Response Surface Methodology: Process and Product Optimization Using Designed Experiments. John Wiley and Sons, New York, NY.

SAS Institute. 2004. SAS/Stat 9.1 User's Guide. Vol. 4. SAS Inst. Inc., Cary, NC.

St-Pierre, N. R. 2000. Invited review: Integrating quantitative findings from multiple studies using mixed model methodology. J. Dairy Sci. 84:741-755.

St-Pierre, N. R., and B. Cobanov. 2006. A model to determine the optimal sampling schedule of diet components. J. Dairy Sci. 90:5383-5394. 

Figure A1. Complete data set for total manure output ( $\mathrm{kg} / \mathrm{cow}$ per day). Trt corresponds to the treatment number of Table 3 . Starch, MP and alfalfa are expressed as per-
centages of diet DM; A, S, and P are the coded variables for alfalfa, starch, and MP, respectively. One unit of A corresponds to $17.68 \%$ of alfalfa (\% of diet DM); 1 unit of S corresponds to $2.83 \%$ starch (\% of diet DM); and 1 unit of $\mathrm{P}$ corresponds to $1.6 \%$ of DM (\% of diet DM). Treatments are centered (coded values of 0 for A, S, and P) at $50 \%$ alfalfa, $26 \%$ starch, and $10.4 \%$ MP. 